\title{
SPECT IMAGING OF BRAIN LESIONS ASSOCIATED WITH REFRACTORY EPILEPSY
}

\author{
N. Deleva, A. Kaprelyan, P. Bochev, A. Klissarova, I. Dimitrov \\ I Clinic of Neurology, Department of Neurology; Department of Radiology and Nuclear medicine, \\ Prof. Paraskev Stoyanov Medical University of Varna
}

Reviewed by: Assoc. prof. B. Balev, PhD

\begin{abstract}
About $30 \%$ of patients with epilepsy have different brain lesions. Development of refractory seizures during the course of the disease requires CT or MRI neuroimaging to detect progression, hemorrhage or tumor recurrence. Although SPECT with different radioligands is not routinely used, numerous studies report its role in the diagnosis of neoplastic and non-neoplastic brain lesions. The aim of this study was to evaluate Tc-99m HMPAO and Tc-99m MIBI uptake in brain lesions associated with intractable seizures. The clinical population included fourteen patients (eight males and six females, mean age $54 \pm 10.6$ ) with refractory epilepsy. Brain SPECT was carried out 20 and 120 minutes after intravenous administration of $740 \mathrm{MBq}(20 \mathrm{mCi})$ Tc-99m HMPAO or Tc-99m MIBI. Refractory simple partial and secondary generalized seizures were observed respectively, in five and nine individuals. EEG showed focus of epileptic activity in all patients. Brain lesions visualized on CT/MRI scans included twelve neoplasms, one arachnoid cyst and one brain abscess. Tc-99m HMPAO SPECT demonstrated region of hypoperfusion in nine patients with gliomas and one with brain abscess. Two cases with high-grade gliomas had an increased Tc-99m MIBI uptake. No radioisotope accumulation was seen postoperatively in a low-grade glioma and also in one benign cystic lesion. Although the mechanisms of epileptogenesis have remained unclear yet, our own results revealed that SPECT neuroimaging may be useful for the precise diagnosis, treatment strategy, and prognosis in patients with brain lesion-associated refractory epilepsy.
\end{abstract}

Keywords: SPECT, Tc-99m HMPAO, Tc-99m MIBI, brain lesions, refractory epilepsy

\section{INTRODUCTION}

About $30 \%$ of patients with epilepsy have different brain lesions, most commonly primary or metastatic tumors $(9,18,26)$. A review of the literature demonstrates that patients with cerebral neoplasms frequently present with epileptic seizures either as an initial symptom or later on $(7,11,15,16)$. The development of intractable seizures during the course of the disease or after neurosurgery can be life threatening and has important clinical and social implications. In accordance to the previous studies, the clinical presentation of refractory epilepsy in patients with brain lesions might be associated with progression, hemorrhage or recurrence $(1,11,13,16)$. Therefore, magnetic resonance imaging (MRI), computed tomography (CT), positron emission tomography (PET) or single-photon emission computed tomography (SPECT) should be performed $(6,10,17)$.

Address for correspondence:

Ara Kaprelyan, Dept. of Neurology,, Medical University Prof. Dr. Paraskev Stoyanov, 55 Marin Drinov St., BG-9002, Varna, BULGARIA

E-mail: arakapri07@yahoo.co.uk
SPECT is a modern diagnostic method for evaluation of regional cerebral blood flow and metabolism in patients with various neurological diseases such as stroke, epilepsy, and dementia $(3,8,12,22)$. Although considered experimental or investigational for the diagnosis of structural brain lesions, numerous preliminary reports indicate that technetium scintigraphy is useful in the assessment of brain tumors, cerebral trauma, vascular malformations, and etc. $(4,10,20)$.

Recently, various radiopharmaceutical agents have been used for detection of different brain lesions $(3,8,12,14)$. Tc-99m HMPAO is a lipophilic agent that freely passes the normal blood-brain barrier and transforms in hydrophilic form that retains in the cells. Therefore, it is a brain perfusion tracer for regional cerebral blood flow with SPECT $(21,23,24)$. Tc-99m MIBI is a similar small lipophilic radioligand that enters cells by diffusion and accumulates significantly more in tumor tissue compared to normal one because of the higher mitochondrial activity in tumor cells. Therefore, the encephalic tracer MIBI has been used to visualize primary, metastatic, and recurrent tumors $(4,12,20,23)$.

Clinical evidence and experience have shown that assessing cerebral function with SPECT is essential in the 
N. Deleva, A. Kaprelyan, P. Bochev ...

diagnosis and management of structural brain lesions associated with refractory epilepsy.

\section{OBJECTIVE}

To evaluate Tc-99m HMPAO and Tc-99m MIBI uptake in brain lesions associated with refractory epilepsy.

\section{MATERIAL AND METHODS}

Fourteen patients ( 8 males and 6 females, mean age 54 $\pm 10.6)$ with different brain lesions and intractable focal seizures with or without secondary generalization were included in the study. Epilepsy was diagnosed according to the Classification of International League Against Epilepsy. Past medical history was revealed and neurological examination performed. Brain SPECT was carried out in all patients 20 and 120 minutes after intravenous administration of $740 \mathrm{MBq}(20 \mathrm{mCi}) \mathrm{Tc}-99 \mathrm{~m}$ HMPAO or Tc-99m MIBI. A single detector camera with a low-energy high-resolution collimator was used for image acquisition. Transverse, sagittal and coronal views were reconstructed and radioligands uptake calculated. EEG findings and CT/MRI images were also evaluated.

\section{RESULTS}

Five patients presented with refractory partial seizures and nine with secondary generalized. EEG demonstrated focus of epileptic activity in all patients. Brain lesions visualized on CT/MRI scans included twelve neoplasms, one arachnoid cyst and one brain abscess. Tc-99m HMPAO SPECT found hypoperfusion in nine patients with gliomas (fig.1), and one with brain abscess (fig.2). Two cases with high-grade gliomas had an increased Tc-99m MIBI uptake.
No radioisotope accumulation was seen postoperatively in a low-grade glioma supposed for a relapse (fig.3) and also in a benign cystic lesion.

\section{DISCUSSION}

Individuals with neoplastic and non-neoplastic brain lesions frequently present with epilepsy $(5,7,15,16,18)$. The management of seizures can be as difficult as the brain tumor, cyst, or abscess itself. Although different etiological factors and underlying mechanisms are discussed, the clinical manifestation of uncontrolled seizures in patients with structural abnormalities supports the idea for possible progression, recurrence, or hemorrhage $(2,5,9,13,16,19)$. Therefore, brain imaging with routine and new functional techniques (PET, SPECT) is recommended to exclude tumor spread or determine possible structural alterations in non-neoplastic lesions $(3,10,13,22,25,26)$. In accordance to these previous studies, we examined fourteen patients with different brain lesions and clinical presentation of refractory epilepsy.

Recently, SPECT with different radioligands has proven useful for the assessment of different brain lesions $(4,12,14,20,23,24)$. According to the literature, the absence of tracer uptake in normal brain, and the favorable physical properties of technetium make the scintigraphic method particularly accurate $(8,12,20,22)$.

Tc-99m HMPAO has been widely used to evaluate regional cerebral blood flow in different cases of brain disease $(2,8,10,12)$. It has been reported to be used in neoplasms, cysts, abscesses, vascular malformations and head trauma $(22,23,24)$. In agreement with our previous results the scans with Tc-99m HMPAO showed regions of hypoperfusion that corresponded to the lesions location and surrounding edema (14). In addition, we suggested that cerebral neoplasms and abscess are able to alter different

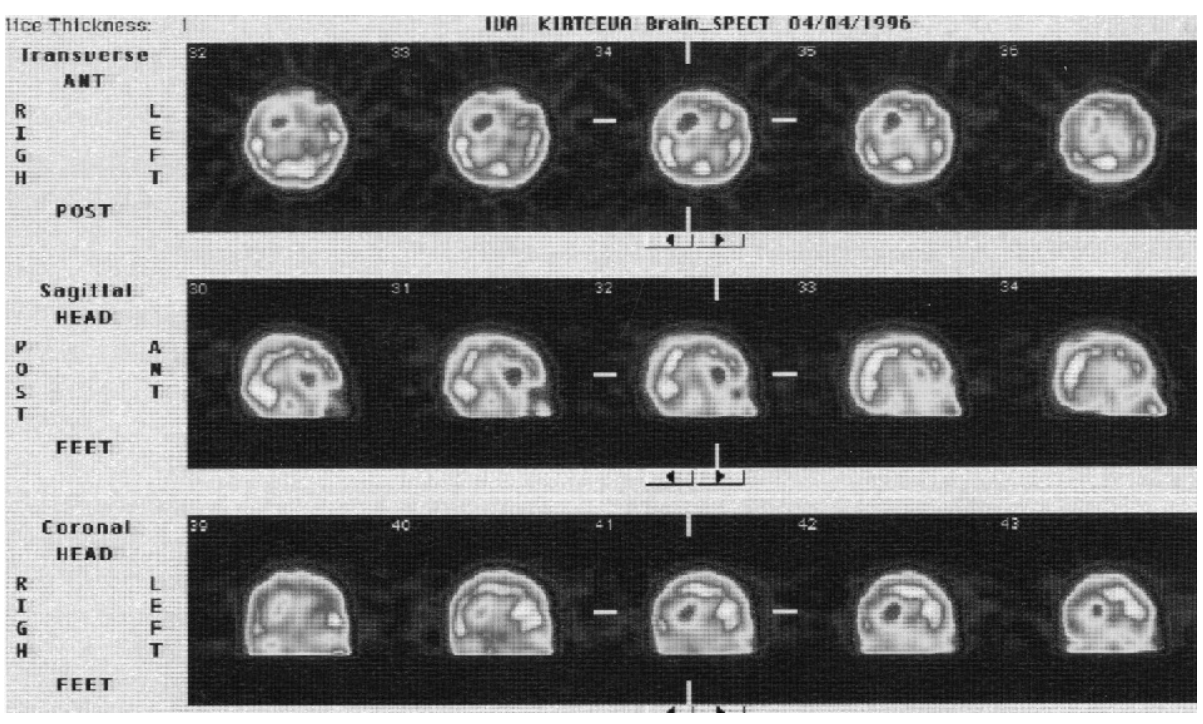

Fig. 1. Tc-99m HMPAO SPECT of 51-years female (I. K.) with fast-growing glioma in right frontal region. 


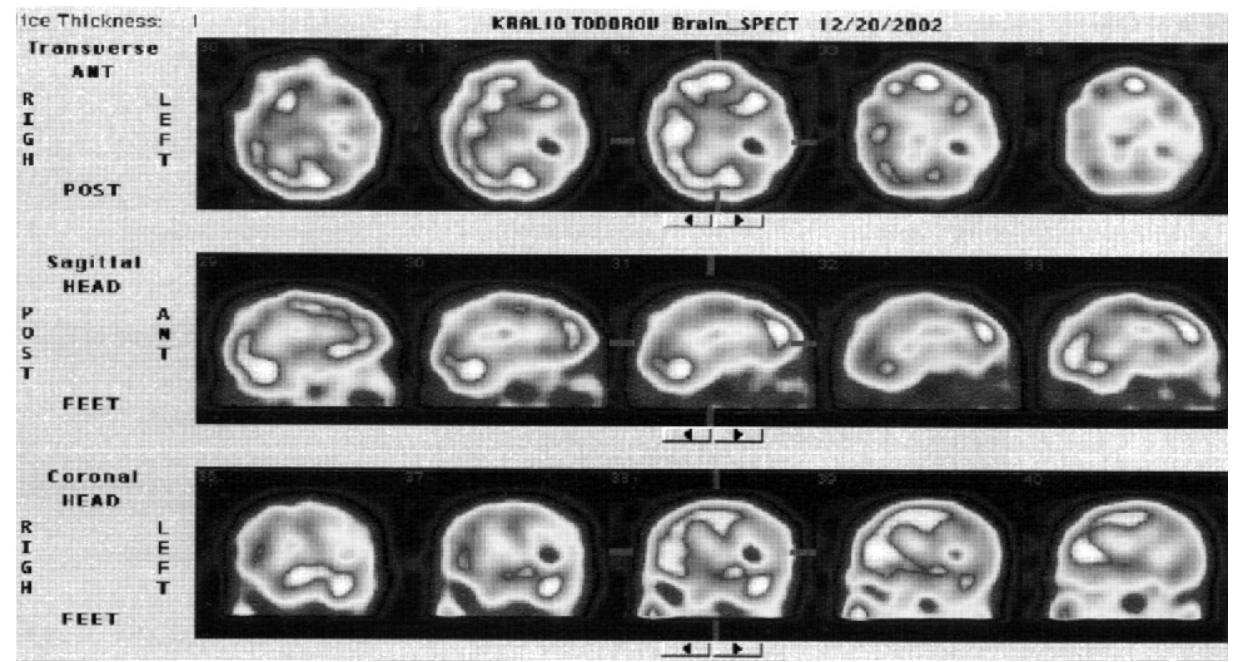

Fig. 2. Tc-99m HMPAO SPECT of 58-years male (K. T.) with brain abscess in left temporo-parietal region.

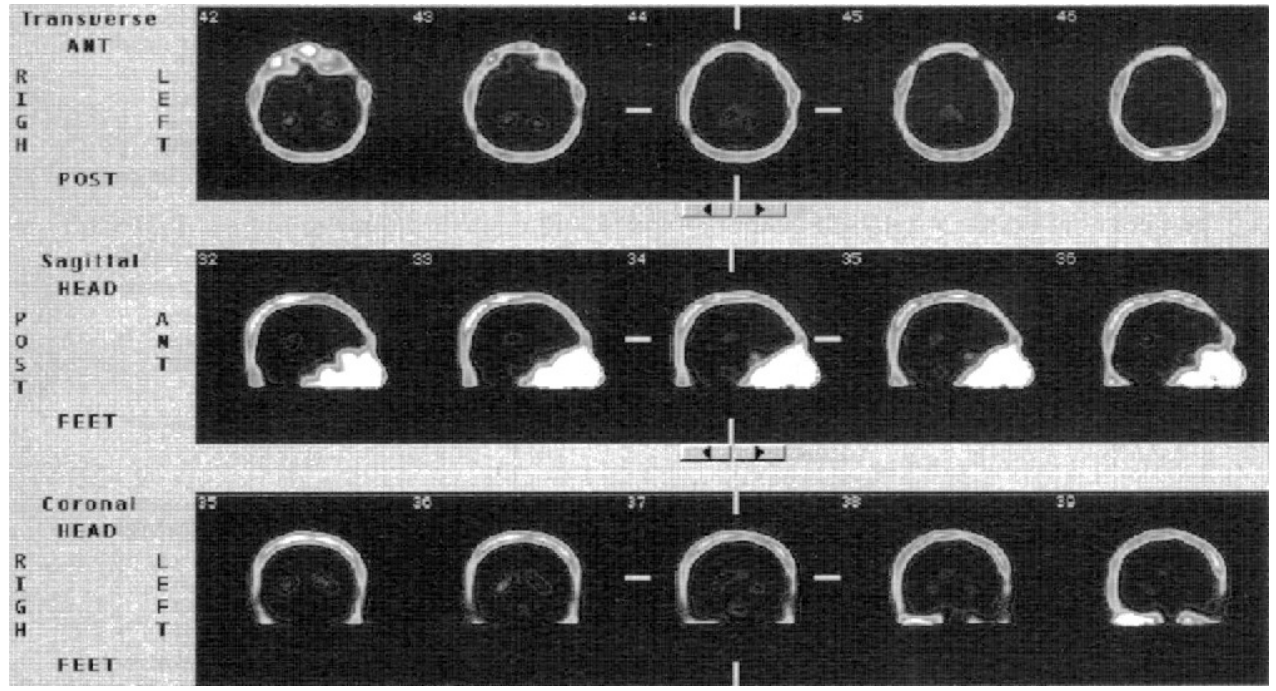

Fig. 3. Tc-99m MIBI SPECT of 58-years female (N. V.) with slow-growing glioma in left fronto-temporal region.

factors that generate epileptic activity and provoke intractable seizures.

Evidence exist that increased vascular supply, disruption of the blood-brain barrier, and tumor cells viability are decisive factors related to increased Tc-99m MIBI uptake in brain tumors $(4,12,17,25)$. In accordance to the previous studies, two of our patients with high-grade gliomas associated with refractory seizures had an increased radioligand accumulation corresponding to the progressive course of the disease $(3,17)$. Regardless of some differences in the uptake indexes, our Tc-99m MIBI SPECT scans demonstrated similar to the literature findings $(4,23,25)$.

Past and current investigations present data that the repeated accumulation of Tc-99m MIBI after surgery corresponds with the potential tumor recurrence $(23,25)$. In the context of uncontrolled seizures observed in a patient with low-grade glioma, we performed a brain scintigraphy. Despite the clinical signs of tumor relapse, SPECT imaging showed no Tc-99m MIBI uptake. The same negative findings were seen in a patient with intractable seizures associated with benign cerebral cyst suspected for neoplastic transformation. Based on these results, we suggested that Tc-99m MIBI SPECT plays an important role for the detection of tumor progression or recurrence in patients with tumor-associated refractory epilepsy.

\section{CONCLUSION}

Although the mechanisms of epileptogenesis have remained unclear yet, we suggested that brain tumors, abscess, and cyst are a common cause for the development of intractable seizures. Tc-99m HMPAO and Tc-99m MIBI SPECT illustrated differences in regional cerebral blood flow and metabolism that corresponded to the type and possible structural alterations of the brain lesions. In addition, the literature review and our own results revealed that SPECT method is useful for the precise diagnosis, treatment strategy, and prognosis in patients with refractory epilepsy and structural brain lesions. 


\section{REFERENCES}

1. Awad I., Rosenfeld J., Ahl J. Intractable epilepsy and structural lesions of the brain: mapping, resection strategies and seizure outcome. Epilepsia, 1991, 32, 179-186.

2. Beaumont A. The pathogenesis of tumour associated epilepsy. Acta Neurochir. (Wien) 1, 2000, 142, $1-15$.

3. Benard F., Romza G., Hustingx R. Imaging gliomas with positron emission tomography and single-photon emission computed tomography. Semin. Nucl. Med., 2 2003, 33, 148-162.

4. Boldari S., Pecorela G., Cosentino S., Minutoli F. Investigation of brain tumours with (99m)Tc-MIBI SPET. Q. J. Nucl. Med., 4, 2002, 46, 336-345.

5. Boon P., Williamson P., Fried I., et al. Intracranial, intraaxial, space-occupying lesions in patients with intractable partial seizures: an anatomoclinical, neuropsychological, and surgical correlation. Epilepsia, 1991, 32, 467-476.

6. Bradley W., Shey R. MR imaging evaluation of seizures. Radiology, 2000, 214, 651-656.

7. Bromfield E., Siddiqui F. EEG in brain tumors. eMedecine, June, 2005.

8. Devous M., Thisted R., Morgan G., et al. SPECT brain imaging in epilepsy: A meta-analysis. J. Nucl. Med., 2, 1998, 39, 285-293.

9. Goh S., McLaren B. About primary brain tumors. Mechanisms uunderlying tumor-associated seizures. BrainScience foundation, 2003.

10. Henry T. Neuroimaging in epilepsy. Contemp. Neurosurg., 2, 1996,18, 1-8.

11. Hildebrand J., Lecaille C., Perennes J., Delattre J. Epileptic seizures during follow-up of patients treated for primary brain tumors. Neurology, 2, 2005, 65, 212-215.

12. Holman B., Abdel-Dayem H. The clinical role of SPECT in patients with brain tumors. J.

Neuroimaging, 5, 1995, Suppl. 1, 34-39.

13. Hwang S. Preoperative and postoperative seizures in patients with astrocytic tumours: analysis of incidence and influencing factors. J. Clin. Neurosci., 5, 2001, 8, 426-429.

14. Kaprelyan A., N. Deleva, A. Klisarova, G. Kuchukov, N. Dimitrov. TC-99m-HMPAO SPECT assessment of rCBF disturbances in patients with brain tumors. Annual proceedings IMAB, 9, 2003, 1, 138-140.
15. Liigant A. Seizure disorders in patients with brain tumors. Eur. Neurol., 1, 2001, 45, 46-51.

16. Mangano F., McBride A., Schneider S. Brain tumors and epilepsy. In: Ettinger A., Devinsky O., eds. Managing epilepsy and co-existing disorders. Boston, Butterworth-Heinemann, 2002, 175-194.

17. Matthews P., Wylezinska M., Cadoux-Hudson T. Novel approaches to imaging brain tumors.Hematology/oncology Cl. N. Am., 15, 2001, 4.

18. Oberndorfer S., Schmal T., Lahrmann H., Urbanits S., Lindner K., Grisold W. The frequency of seizures in patients with primary brain tumors or cerebral metastases. Wien. Klin. Wochenschr., 2002, 114, 911-916.

19. Ray S., Kundu B., Kundu S., Roy S., Sharma $\mathrm{S}$. Distinguishing neoplastic and non-neoplastic ring enhancing lesions of the brain detected in CT and /or MRI with the help of correlative nuclear imaging. Ind. J. Radiol. Imag., 2, 2002, 12, 189-195.

20. Single Photon Emission Computed Tomography (SPECT).Clinical Policy Bulletins, 0376, 2006.

21. Sjoholm H, Elmquist D., Rehncrona S., Rosen I., Salford L. SPECT imaging of gliomas with Thallium-201 and Technetium-99m-HMPAO. Acta Neurol. Scand., 1 1995, 91, 66-70.

22. Sperling M. Neuroimaging in epilepsy: recent developments in MR imaging, positron emission tomography, and single-photon emission tomography. Neurol. Clin. 1993, 11, 883-903.

23. Sygitowicz M., Lass P., Lyczak P., Stepien-Kocmiel E., Taraszewska M., Bandurski T. Tc-99m-MIBI and Tc-99m-HMPAO accumulation in primary and metastatic brain tumors assessed by brain SPECT. Neurol. Neurochir. Pol., 5, 1998, 32, 1099-1106.

24. Terada H., Kamata N. Contribution of the combination of (201) T1 SPECT and (99m) T(c) SPECT to the differential diagnosis of brain tumors and tumor-like lesions. A preliminary report. J.

Neuroradiol., 2, 2003, 30, 91-94.

25. Jeune F., Dubois F., Blond S., Steinling M. Sestamibi technetium-99m brain single-photon emission computed tomography to identify recurrent glioma in adults: 201 studies. J. Neurooncol., 2, 2006, 77, 177-183.

26. Wen P., Marks P. Medical management of patients with brain tumors. Curr. Opin. Oncol., 3, 2002, 14, 299-307. 